\title{
APLICAÇÃO DOS TESTES DE PADRÃO TEMPORAL EM CRIANÇAS COM GAGUEIRA DESENVOLVIMENTAL PERSISTENTE
}

\author{
Application of temporal pattern tests in children \\ with persistent developmental stuttering
}

Rosimeire da Silva ${ }^{(1)}$, Cristiane Moço Canhetti de Oliveira ${ }^{(2)}$, Ana Cláudia Vieira Cardoso ${ }^{(3)}$

\begin{abstract}
RESUMO
Objetivo: caracterizar e comparar o desempenho das crianças com diagnóstico de gagueira nos testes de padrão temporal, com crianças sem queixas e/ou sinais de transtornos psiquiátricos ou neurológicos, dificuldades de fala, audição, linguagem e/ou aprendizagem. Método: participaram 30 crianças entre 9 e 12 anos de idade, de ambos os gêneros, divididas em dois grupos: GI - 15 crianças com gagueira desenvolvimental persistente; GII - 15 crianças sem queixas e/ou sinais de transtornos psiquiátricos ou neurológicos, dificuldades de fala, audição, linguagem e/ou aprendizagem. Para avaliação do processamento auditivo temporal, foi aplicado os Testes Tonais de Padrão de Frequência (PPS-Pitch Pattern Sequence Test) e Testes Tonais de Padrão de Duração (DPS - Duration Pattern Sequence Test). Resultados: o grupo II apresentou desempenho superior no teste de padrão de frequência e de padrão de duração quando comparado ao grupo I. Os resultados indicaram que houve diferença estatisticamente significante entre os grupos estudados. Conclusão: os participantes do grupo I apresentaram desempenho alterado nos testes de padrão temporal, o que indica que existe relação entre a gagueira e o transtorno do processamento auditivo.
\end{abstract}

DESCRITORES: Audição; Gagueira; Testes Auditivos

\section{INTRODUÇÃO}

Gagueira desenvolvimental persistente (GDP) é um transtorno da fluência que se manifesta por rupturas involuntárias no fluxo da fala, que se inicia na infância e que pode persistir na fase adulta ${ }^{1-4}$. Este distúrbio é caracterizado por excessiva

(') Fonoaudióloga; Especializanda do Curso de Pós-Graduação Lato Sensu em Linguagem da Faculdade de Filosofia e Ciências da Universidade Estadual Paulista Júlio de Mesquita Filho - UNESP, Marília,SP, Brasil.

(2) Fonoaudióloga; Docente do Departamento de Fonoaudiologia da Faculdade de Filosofia e Ciências da Universidade Estadual Paulista FFC/ UNESP, Marília, SP; Doutora em Ciências Biológicas (Genética) pela Universidade Estadual Paulista Júlio de Mesquita Filho- UNESP, Botucatu,SP, Brasil.

(3) Fonoaudióloga; Docente do Departamento de Fonoaudiologia da Faculdade de Filosofia e Ciências da Universidade Estadual Paulista FFC/ UNESP, Marília, SP; Doutora em Distúrbios da Comunicação Humana pela Universidade Federal de São Paulo - UNIFESP, São Paulo, SP.

Conflito de interesses: inexistente repetição de sons, de sílabas e palavras monossilábicas, prolongamentos e bloqueios, geralmente em mais de $3 \%$ da fala ${ }^{5,6}$.

Embora a etiologia da gagueira ainda não tenha sido precisamente identificada, as pesquisas atuais têm mostrado que fatores genéticos estão envolvidos na sua transmissão ${ }^{7}$. Acredita-se que os fatores genéticos podem afetar pequenas e sutis mudanças na estrutura e função do cérebro.

Gagueira é multidimensional ${ }^{6}$, na qual inúmeros fatores, como biológicos, psicológicos e sociais interagem de forma complexa, influenciando o desenvolvimento pessoal e as interações sociais do indivíduo que gagueja. Dentre os fatores biológicos, destacam-se as habilidades auditivas como um fator que influencia na fluência da fala, pois estudos mostraram a associação entre gagueira e processamento auditivo ${ }^{8-10}$ e o uso da retroalimentação auditiva atrasada (RAA) no tratamento da gagueira ${ }^{11}$. 
Como gagueira é um distúrbio da fluência, é importante compreender sobre a fluência. Os segmentos da fala na emissão fluente ou contínua são coarticulados, ou seja, seu padrão acústico é modificado em relação à duração, à intensidade e à frequência. Para que a fala seja fluente, dois sistemas operacionais neurais, o simbólico e o de sinais, devem estar temporalmente equilibrados, antes que a mensagem gerada chegue ao córtex motor $^{12}$. O sistema de sinas determina a duração proporcional da sílaba na palavra e a ordem de sequencialização ordenada dos espaços fonéticos. Quando esses dois sistemas operam em equilíbrio - segmentação e ordenação - a fala é gerada sem rupturas. Quando os sistemas estão em desequilíbrio o fluxo da fala será temporariamente rompido, gerando as disfluências ${ }^{12}$.

Portanto, a maneira como os falantes processam as informações auditivas principalmente relacionadas à percepção da fala, tornam-se fundamentais para compreender possíveis dificuldades apresentadas na linguagem expressiva, entre elas a gagueira.

Estudos referem que os processos neuroaudiológicos envolvidos no processamento da fluência podem ser investigados por meio da avaliação do processamento auditivo ${ }^{8}$.

O processamento auditivo (central) $(\mathrm{PA}(\mathrm{C}))$ refere-se à eficiência e a efetividade com que o sistema nervoso central (SNC) utiliza a informação auditiva $^{13}$. A avaliação do processamento auditivo verifica como o indivíduo recebe as informações acústicas utilizando habilidades auditivas que são essenciais para ele apreender o que ouve ${ }^{14}$.

As habilidades do processamento temporal podem ser consideradas como a base do processamento auditivo, especificamente no que concerne à percepção de fala ${ }^{15}$. Esta afirmação é fundamentada no fato de que muitas das características da informação auditiva são de alguma forma, influenciadas pelo tempo ${ }^{15,16}$.

A imprecisão temporal na percepção de fala pode levar a momentos de disfluência, já que a fala fluente ocorre a partir da interação dos aspectos acústicos, e a diminuição das habilidades de processamento pode estar relacionada à incapacidade de manutenção da fala fluente ${ }^{17}$. Portanto, o processamento auditivo pode ser um dos fatores que contribuem para a produção do discurso disfluente no nível da produção da sílaba, já que os gagos como um grupo apresentam evidências de distúrbio do processamento auditivo?.

Uma das categorias do processamento auditivo temporal é a ordenação ou sequencialização temporal, que se refere ao processamento de dois ou mais estímulos auditivos na sua ordem de ocorrência no tempo ${ }^{15,18 .}$ A resolução temporal ou discriminação foi definida como a habilidade do sistema auditivo em detectar mudanças rápidas no estímulo sonoro ou o menor intervalo de tempo para discriminar entre dois estímulos sonoros ${ }^{19}$.

$\mathrm{Na}$ prática clinica, a ordenação temporal é freqüentemente avaliada por meio dos testes de padrão de freqüência e duração, que têm por objetivo fazer com que o ouvinte reconheça contornos acústicos ${ }^{15}$. Diversos processos acústicos contribuem para essa habilidade, incluindo a discriminação de diferentes estímulos auditivos; sequencialização dos elementos lingüísticos; ou ordenação temporal de sons; reconhecimento do todo; transferência inter hemisférica e memória quanto ao número de itens utilizados na série ${ }^{20}$.

A percepção adequada da duração, enquanto sequência de eventos é imprescindível para o processamento das pistas acústicas da fala ${ }^{20}$. $\mathrm{O}$ tipo de prejuízo envolvido no processamento auditivo temporal é denominado gnósico não-verbal que designam alterações nos testes de padrão temporal ou de reconhecimento de sons não-verbais em escuta dicótica. Dificuldade de adquirir ou armazenar informações que se sucedem no tempo ${ }^{21}$, e esta dificuldade pode colaborar para a produção de uma fala disfluente.

A habilidade de ordenação temporal de frequência e de duração é utilizada principalmente para análise dos aspectos de prosódias da fala como ritmo, acentuação e entoação ${ }^{22}$.

Uma vez que os indivíduos que apresentam processamento auditivo alterado não possuem uma discriminação de frequência, intensidade e duração sonora adequadas, a prosódia e a entonação do que é falado provavelmente não são apreciadas por completo ${ }^{23}$.

Com base no exposto acima, os objetivos deste estudo foram caracterizar e comparar o desempenho das crianças com diagnóstico de gagueira desenvolvimental persistente nos testes de padrão temporal, com crianças sem queixas e/ ou sinais de transtornos psiquiátricos ou neurológicos, dificuldades de fala, audição, linguagem e/ou aprendizagem.

\section{MÉTODO}

Participaram deste estudo clínico com corte transversal 30 crianças na faixa etária de 9 a 12 anos de idade, de ambos os gêneros, divididos da seguinte forma:

Grupo I (GI) constituído por 15 crianças do Estágio Supervisionado de Distúrbios da Fluência do Centro de Estudos da Saúde e Educação CEES//UNESP-Marília com diagnóstico de gagueira 
desenvolvimental persistente (GDP), sendo nove $(60 \%)$ do gênero masculino e seis $(40 \%)$ do gênero feminino, com idade média de 10,2 anos. Os requisitos de inclusão dos participantes foram:

- idade entre 9 e 12 anos: uma vez que a idade e o efeito da neuromaturação da via auditiva podem interferir com os resultados da avaliação comportamental do processamento auditivo ${ }^{24}$;

- ser falante nativo do português brasileiro, e;

- apresentar queixa de gagueira por parte dos pais ou familiares.

Para GI, o diagnóstico de gagueira desenvolvimental persistente (GDP) foi estabelecido segundo os critérios:

- início dos sintomas nos anos pré-escolares;

- duração das disfluências por no mínimo 1 ano;

- presença de no mínimo 3\% de disfluências gagas (repetições de palavras monossilábicas, de sílabas ou de sons; prolongamentos e bloqueios)

- receber 11 pontos ou mais (critério mínimo para a gravidade da gagueira ser classificada como "leve") no Stuttering Severity Instrument - 3 $(\mathrm{SSI}-3)^{25}$.

Grupo II (GII) composto por 15 crianças que passaram por triagem fonoaudiológica no Centro de Estudos da Saúde e Educação - CEES/UNESPMarília e após esta nenhum dos participantes apresentou sinais de transtornos psiquiátricos ou neurológicos, dificuldades de fala, audição, linguagem e/ou aprendizagem. Sendo 12 (80\%) do gênero masculino e $3(20 \%)$ do gênero feminino, com idade média de 9,8 anos. Este foi considerado como grupo controle.

Os critérios de exclusão dos participantes foram: apresentar qualquer distúrbio neurológico genético ou não, tais como distonia, doenças extrapiramidais, deficiência mental, epilepsia, transtorno do déficit de atenção e hiperatividade (TDAH); sintomas ou condições psiquiátricas; apresentar alterações de comunicação oral não compatíveis com a idade (com exceção à gagueira para GI); apresentar perda auditiva condutiva ou neurossensorial, e; outras condições pertinentes que poderiam gerar erros no diagnóstico.

Neste estudo foram realizados os seguintes procedimentos:

- Termo de consentimento pós-informado: Conforme resolução do Conselho Nacional de Saúde CNS 196/96, anteriormente ao início das avaliações, os pais ou responsáveis dos pacientes selecionados assinaram o termo de consentimento pós- informado para autorização da realização do estudo.

- Avaliação da Fluência: Uma amostra de fala espontânea das crianças contendo 200 sílabas fluentes foi filmada, transcrita e analisada para determinar a porcentagem de disfluências gagas e a presença ou ausência de gagueira. $O$ Instrumento de Gravidade da Gagueira (SSI-3) ${ }^{25}$ foi utilizado para cada participante do GI classificando a gagueira em leve, moderada, grave ou muito grave. Este teste avaliou a frequência e duração das interrupções da fala, assim como a presença de concomitantes físicos associados às disfluências, seguindo protocolo proposto por Riley (1994) ${ }^{25}$.

- Avaliação audiológica: A avaliação da audição periférica e a avaliação do processamento auditivo temporal foram realizadas em cabine acústica com audiômetro GSI 61, calibrado de acordo com a norma ISO 8253-1 (1989) por via aérea em fones auriculares TDH-50. Os participantes foram submetidos à anamnese, meatoscopia, audiometria tonal limiar, logoaudiometria, timpanometria e pesquisa de reflexos acústicos ispsilaterais e contralaterias, por meio do imitanciômetro GSI-38, a fim de descartar presença de perda auditiva periférica e alterações de orelha média. Dessa forma todas as crianças da pesquisa apresentaram limiares audiométricos dentro dos padrões de normalidade, ou seja, 15 dBNA (Decibel Nível de Audição) ${ }^{26}$ e curva timpanométrica do tipo $A^{27}$.

- Avaliação do processamento auditivo temporal: $O$ teste de Padrão de Freqüência e o teste de Padrão de Duração, gravados em $C D$, foram aplicados por meio de $C D$ player da marca Cobby acoplado ao audiômetro GSI-61. Foram apresentados estímulos sonoros de forma binaural a 50 dBNS (Decibel Nível de Sensação) acima da média dos limiares tonais nas freqüências de 500, 1000 e $2000 \mathrm{~Hz}$ de cada participante.

No teste de Padrão de Frequência foram apresentadas 30 sequências de padrões, formadas por três tons que diferem entre si em duas frequências: $1430 \mathrm{~Hz}(\mathrm{~A})$ e $880 \mathrm{~Hz}(\mathrm{G})$. Cada tom tem a duração de $500 \mathrm{~ms}$, com diferença de $10 \mathrm{~ms}$. O intervalo entre os três tons é de $300 \mathrm{~ms}$ e o intervalo entre cada sequência de tons é de $10 \mathrm{seg}$. Os tons são combinados em seis diferentes padrões de frequência: AAG, AGG, AGG, GAA, GAG e GGA. $O$ indivíduo foi orientado a nomear os padrões ouvidos utilizando o termo "pi" para o tom agudo e "pó" para o grave ${ }^{28}$.

O teste de Padrão de Duração foi aplicado a partir da apresentação de 30 sequências de padrões, cada sequência composta por três tons puros de $1 \mathrm{kHz}$, que se diferenciam quanto à duração: longo (L) e curto (C). O tom longo dura $500 \mathrm{~ms}$ e o tom curto $250 \mathrm{~ms}$. O intervalo entre os tons é de $300 \mathrm{~ms}$. 
São seis possibilidades de sequências: LLC; LCL; LCC; CLL; CLC e CCL. O indivíduo foi orientado a imitar ou murmurar o padrão apresentado ${ }^{28}$.

Este estudo foi realizado após aprovação do Comitê de Ética em Pesquisa da Faculdade de Filosofia e Ciências da Universidade Estadual Paulista - CEP/FFC/UNESP/ Marília-SP sob o protocolo 1739/2009.

Para a realização da análise estatística, foi utilizado o programa SPSS (Statistical Package for Social Sciences) em sua versão 17.0 , com nível de significância de 5\% (0,050). Foram aplicados o Teste Exato de Fisher e o Teste de Mann-Whitney, com o intuito de verificar possíveis diferenças entre ambos os grupos estudados.

\section{RESULTADOS}

Os resultados apresentados na tabela 1, por meio da análise estatística realizada com o Teste de Mann-Whitney, representam o número médio de acertos dos grupos estudados nos testes de padrão de frequência e de duração. Como pode ser observado houve diferença estatisticamente significante entre os grupos estudados para as variáveis de interesse (teste de padrão de freqüência, $p$-valor $=0$, 005; e teste de padrão de duração, $p$-valor $<0,001)$. $\mathrm{O}$ grupo de crianças com gagueira (G I) apresentou maior variabilidade nas respostas dos testes de padrão de freqüência e de duração quando comparado ao grupo controle (G II).

O desempenho no teste de padrão de freqüência das crianças com gagueira (grupo I) e crianças controles (grupo II) foi comparado pelo teste Exato de Fisher (Tabela 2). Os dados demonstraram diferença estatisticamente significante entre os grupos $(p$-valor $=0,001)$.

A distribuição dos participantes de acordo com o desempenho do teste de padrão de duração está demonstrada na tabela 3 . Os resultados indicaram que os grupos se diferenciaram estatisticamente no teste Exato de Fisher ( $p$-valor < 0, 001).

Tabela 1 - Distribuição dos participantes segundo o número de acertos nos testes de padrão temporal

\begin{tabular}{lcccccccc}
\hline Variável & Grupo & $\mathbf{n}$ & Média & $\begin{array}{c}\text { Desvio- } \\
\text { padrão }\end{array}$ & Mínimo & Máximo & Mediana & Sig. (p) \\
\hline \multirow{3}{*}{ PPS_perc } & I & 15 & 77,07 & 14,29 & 47,00 & 100,00 & 73,00 & $0,005^{\star}$ \\
& II & 15 & 91,00 & 6,04 & 85,00 & 100,00 & 90,00 & \\
\hline \multirow{2}{*}{ DPS_perc } & Total & 30 & 84,03 & 12,90 & 47,00 & 100,00 & 85,00 & \\
& II & 15 & 50,47 & 18,11 & 27,00 & 80,00 & 47,00 & \\
& Total & 15 & 86,60 & 3,07 & 83,00 & 90,00 & 85,00 & $<0,001^{*}$ \\
\hline
\end{tabular}

${ }^{*}$ Teste de Mann-Whitney $(\mathrm{p} \leq 0,05)$

Legenda: PPS_perc: Percentual do teste de padrão de frequência; DPS_perc: Percentual do teste de padrão de duração.

Tabela 2 - Distribuição dos participantes de acordo com o desempenho no teste de padrão de frequência

\begin{tabular}{|c|c|c|c|c|}
\hline \multirow{2}{*}{ GRUPO } & \multicolumn{2}{|c|}{ Class_PPS } & \multirow{2}{*}{ Total } & \multirow{2}{*}{ Sig. (p) } \\
\hline & normal & alterado & & \\
\hline \multirow{2}{*}{ I } & 6 & 9 & 15 & \multirow{4}{*}{$p=0,001$} \\
\hline & $40,00 \%$ & $60,00 \%$ & $100,00 \%$ & \\
\hline \multirow{2}{*}{ II } & 15 & 0 & 15 & \\
\hline & $100,00 \%$ & $0,00 \%$ & $100,00 \%$ & \\
\hline \multirow{2}{*}{ Total } & 21 & 9 & 30 & \\
\hline & $70,00 \%$ & $30,00 \%$ & $100,00 \%$ & \\
\hline
\end{tabular}

*Teste Exato de Fisher $(p \leq 0,05)$

Legenda: Class_PPS: Classificação do teste de padrão de frequência. 
Tabela 3 - Distribuição dos participantes de acordo com o desempenho no teste de padrão duração

\begin{tabular}{|c|c|c|c|c|}
\hline \multirow{2}{*}{ GRUPO } & \multicolumn{2}{|c|}{ Class_DPS } & \multirow{2}{*}{ Total } & \multirow{2}{*}{ Sig. (p) } \\
\hline & normal & alterado & & \\
\hline \multirow{2}{*}{ I } & 0 & 15 & 15 & \multirow{4}{*}{$\mathrm{p}<0,001^{*}$} \\
\hline & $0,00 \%$ & $100,00 \%$ & $100,00 \%$ & \\
\hline \multirow{2}{*}{ II } & 15 & 0 & 15 & \\
\hline & $100,00 \%$ & $0,00 \%$ & $100,00 \%$ & \\
\hline \multirow{2}{*}{ Total } & 15 & 15 & 30 & \\
\hline & $50,00 \%$ & $50,00 \%$ & $100,00 \%$ & \\
\hline
\end{tabular}

${ }^{*}$ Teste Exato de Fisher $(p \leq 0,05)$

Legenda: Class_DPS: Classificação do teste de padrão de duração.

\section{DISCUSSÃO}

As respostas dos testes aplicados nesta pesquisa são de grande importância na avaliação do processamento auditivo, tendo em vista que as habilidades do processamento temporal podem ser consideradas como a base do processamento auditivo ${ }^{15}$. Considerando o fato de o processamento auditivo temporal estar intimamente relacionada à percepção da fala, passa a ser de extrema importância a aplicação de testes envolvendo habilidades temporais em indivíduos com queixas relacionadas à fluência da fala ${ }^{15}$.

Por meio da análise dos resultados obtidos neste estudo, verificou-se que, o grupo de crianças com gagueira (grupo I) apresentou alteração no desempenho nos testes de padrão temporal quando comparadas às crianças do grupo controle (grupo II), o que corrobora com a literatura que afirma que os gagos como um grupo apresentam evidências de distúrbio do processamento auditivo ${ }^{8-10}$. Os achados observados neste estudo foram semelhantes aos de outros estudos encontrados na literatura, que encontraram alteração na avaliação comportamental do PA em mais de $90 \%$ dos indivíduos gagos ${ }^{8,9}$.

O comprometimento rítmico característico da gagueira $^{29}$, e a alteração nos processos suprasegmentais da fala podem estar relacionados ao transtorno do processamento auditivo nos testes temporais encontrado no grupo I. Portanto, estes dados mostram a associação entre gagueira e transtorno do processamento auditivo e apontam para a importância da inclusão dos testes de processamento auditivo no processo diagnóstico dos distúrbios da fluência, como destacado anteriormente na literatura ${ }^{9}$.

A alteração do processamento auditivo em crianças disfluentes pode ser considerada como um fator de risco para o desenvolvimento da gagueira, auxiliando assim os fonoaudiólogos na difícil tarefa da determinação do risco que uma criança disfluente apresenta para o desenvolvimento de uma gagueira crônica.

Portanto, os achados desta pesquisa reforçam que a gagueira é multifatorial, conforme descrito na literatura ${ }^{6}$, e ressalta que as habilidades auditivas devem ser consideradas juntamente com as habilidades de fala, o ambiente comunicativo, a consciência do distúrbio, os sentimentos e as atitudes em relação à gagueira tanto na avaliação como na terapia.

O transtorno do processamento auditivo encontrado neste estudo que avaliou o processamento temporal em crianças com gagueira foi alteração no processo gnósico não verbal resultante de um desempenho inferior nos testes aplicados, conforme descrito previamente ${ }^{21}$.

O grupo I (crianças com gagueira) apresentou diferença estatisticamente significante nos dois testes aplicados quando comparados com o grupo II (controle). Os resultados observados mostraram alterações em $100 \%$ do grupo de crianças com gagueira desenvolvimental persistente (grupo I) no teste de padrão de duração e em $60 \%$ no teste de padrão de freqüência. Verifica-se, portanto, maior ocorrência de resultados alterados no teste de padrão de duração, quando comparado com o teste de padrão de freqüência. Não foram encontrados estudos na literatura compilada que relatassem resultados da aplicação dos testes temporais em crianças com gagueira.

O parâmetro acústico de duração é uma medida objetiva que compõe a análise acústica tanto da voz quanto da fala e está diretamente relacionado com o ritmo e a taxa de elocução ${ }^{10}$. Dessa forma, notamos que as crianças com gagueira apresentaram uma grande inabilidade ao lidar com estímulos 
não-verbais, mostrando, assim, dificuldade para extrair os aspectos supra-segmentares da fala. Baseados em nossa experiência com gagueira, destacamos a importância do trabalho com as habilidades do PA no processo terapêutico de crianças gagas, tendo em vista que uma das técnicas mais trabalhadas é a redução da taxa da elocução ou prolongamento das vogais ${ }^{30}$. Acreditamos que o trabalho com as habilidades do padrão temporal do PA irá propiciar resultados mais eficazes na terapia da gagueira.Essa relação entre os processos temporais e a percepção da fala marca as perturbações da temporalidade no controle motor da fala presentes no discurso fluente e disfluente do indivíduo que manifesta a gagueira ${ }^{4}$.

Com base nos achados desse trabalho acreditase que a diferença estatisticamente significante encontrada nos resultados do teste de padrão temporal das crianças com gagueira em relação às crianças do grupo controle seja um indicador da inabilidade de processar dois ou mais estímulos auditivos na sua ordem de ocorrência no tempo. E esta inabilidade pode colaborar para a dificuldade que freqüentemente as pessoas com gagueira apresentam em transferir e manter a fala fluente para situações fora da sala de terapia. No ambiente terapêutico as informações auditivas são apresentadas geralmente num ambiente mais silencioso, com pouco ruído, facilitando, portanto a realização de figura-fundo auditiva e consequentemente a fala fluente. Diferentemente do ambiente extra-clínica que frequentemente apresenta ruídos auditivos, prejudicando a percepção da fala.

Enfatiza-se a necessidade de mais pesquisas sobre este tema, visto que a gagueira é um distúrbio complexo e são inúmeras as diferentes habilidades do processamento auditivo.

\section{CONCLUSÃO}

Com base nos resultados deste estudo concluímos que crianças com gagueira desenvolvimental persistente (grupo I) apresentaram desempenho alterado nos testes de padrão temporal (de freqüência e duração), com diferença estatisticamente significante em relação ao grupo controle (grupo II).

Este estudo contribui para salientar a importância da inclusão da avaliação do teste do processamento temporal no processo diagnóstico da gagueira. Além disso, ressalta a necessidade de acrescentar o trabalho com as habilidades auditivas na intervenção terapêutica para favorecer a aquisição, manutenção e transferência da fluência pelas crianças com gagueira.

\begin{abstract}
Purpose: to characterize and compare the performance of children with diagnosis of stuttering under temporal pattern tests with the performance of children without complaints. Method: we evaluated 30 children between 9 and 12-year old, both genders, divided in two groups: Gl - 15 children with persistent development stuttering; GII - 15 children without complaints and/or signals of psychiatric or neurological upheavals, speech, hearing, language and/or learning difficulties. To evaluate the auditory processing we applied Pitch Pattern Sequence Test (PPS) and Duration Pattern Sequence Test (DPS). Results: group II showed a better performance on PPS and DPS tests when compared with group I. The results indicated that there was a significant statistical difference between the two groups. Conclusion: the subject on group I showed a bad performance on the tests applied, indicating that there is a relation between stuttering and auditory processing disorder.
\end{abstract}

KEYWORDS: Hearing; Stuttering; Hearing Tests

\section{REFERÊNCIAS}

1. Logan KJ, Mullins MS, Jones KM. The depiction of stuttering in contemporary juvenile fiction: implications for clinical practice. Psychol schools, 2008; 45(7):609-26.

2. Andrade CRF, Sassi FC, Juste F, Mendonça LIZ. Persistente developmental stuttering as a cortical-subcortical dysfuncition: evidence from muscle activation. Arq Neuropsiquiatr 2008;66(3-B):659-64.

3. Chang S, Synnestvedt A, Ostuni J, Ludlow CL. Similarities in speech and white matter characteristics in idiopathic developmental stuttering and adult-onset stuttering. J Neuroling.2009;23 (5): 455-69. 
4. Arcuri CF, Osborn E, Schiefer AM, Chiari BM. Taxa de elocução de fala segundo a gravidade da gagueira. Pró-Fono: Rev Atual Cient. 2009; 21(1): 45-50.

5. Sawyer J, Yairi E. The effect of sample size on the assessment of stuttering severity. American. J Speech Lang Pathol. 2006;15(1):36-44.

6. Wittke-Thompson JK, Ambrose N, Yairi EC, Cook EH, Ober C. et al. Genetic studies of stuttering in a founder population. J Fluency Disord. 2007;32:33-50.

7. Kang C, Riazuddin S, Mundorff J, Krasnewich D, Friedman, P.; Mullikin, J.C.; Drayna, D. Mutations in the lysosomal enzyme-targeting pathway and persistent stuttering. N Engl J Med. 2010; 362(8): 677-85.

8. Schiefer AM, Barbosa LMG, Pereira LD. Considerações preliminares entre uma possível correlação entre gagueira e os aspectos lingüísticos e auditivos. Pró-Fono: Rev Atual Cient. 1999; 11(1): 31-7. 9. Andrade NA, Gil D, Schiefer AM, Pereira LD. Avaliação comportamental do processamento auditivo em indivíduos gagos. Pró-fono: Rev Atual Cient. 2008; 20(1): 43-8.

10. Andrade NA, Gil D, Schiefer AM, Pereira LD. Processamento auditivo em gagos: análise do desempenho das orelhas direita e esquerda. Rev Soc Bras Fonoaudiol. 2008; 13(1): 20-9.

11. Van Borsel J, Sierens S, Pereira MMB. Realimentação auditiva atrasada e tratamento de gagueira: evidências a serem consideradas. Pró-Fono Rev Atual Cient. 2007; 19(3): 323-32.

12. Andrade CRF. Processamento da Fala Aspectos da Fluência. Pró-Fono Rev Atual Cient. 2000; 12(1): 69-71.

13. Nascimento FM, Monteiro RAM, Soares CD, Ferreira MIDC. Habilidades de Sequencialização Temporal em Músicos Violinistas e Não-Músicos. Arq. Int. Otorrinolaringol./Intl. Arch. Otorhinolaryngol. 2010; 14(2): 217-24.

14. Liporaci FD; Frota SMMC. Envelhecimento e ordenação temporal auditiva. Rev CEFAC. No prelo 2010.

15. Samelli AG, Schochat E. The gaps-in-noise test: gap detection thresholds in normal-hearing young adults. Int J Audiol. 2008; 47(5): 238-45.

http://dx.doi.org/10.1590/S1516-18462011005000045

RECEBIDO EM: 30/06/2010

ACEITO EM: 29/11/2010

Endereço para correspondência:

Rosimeire da Silva

Rua Dona Julia Nomura, 132

Marília-SP

CEP: $17501-300$

E-mail: meirefono@gmail.com
16. Smith NA, Trainor LJ, Shore DI. The development of temporal resolution: between-channel gap detection in infants and adults. J Speech Lang Hear Res. 2006; 49(5): 1104-13.

17. Meyers SC, Hughes LF, Schoeny, Z. G.Temporal-phonemic processing skills in adult stutterers and nonstutterers. J Speech Lang Hear Res. 1989; 32(2): 274-80.

18. Balen SA, Massignani R, Schillo R. Aplicabilidade do software fast forword na reabilitação dos distúrbios do processamento auditivo: resultados iniciais. Rev CEFAC. 2008; 10(4): 572-87.

19. Zaidan E, Garcia AP, Tedesco MLF, Baran JA. Desempenho de adultos jovens normais em dois testes de resolução temporal. Pró-fono: Rev Atual Cient. 2008; 20 (1): 19-24.

20. Musiek FE, Baran JA, Pinheiro ML. Neuroaudiology: case studies. San Diego: Singular; 1994.

21. Alvarez AMMA, Balen SA, Misorelli MIL, Sanchez ML. Processamento auditivo central: proposta de avaliação e diagnóstico diferencial. In: Munhoz MSL, Capovilla HH, Silva MLG, Ganança MM, editores. Audiologia clínica. São Paulo: Atheneu; 2000. p. 103-20.

22. Balen SA, Bretzke L, Mottecy CM, Liebel G, Boeno MRM, Gondim LMA. Temporal resolution in children: comparing normal hearing, conductive hearing loss and auditory processing disorders. Int J Pediatr Otolaryngol. 2009; 5 (1):123-9.

23. Thompson ME, Abel SM. Indices of hearing in patients with central auditory pathology II: choice response time. Scand Audiol Suppl. 1992; 35:17-22.

24. Musiek FE, Gollege KM, Lamb LE, Lamb P. Select issues in screening for central auditory processing dysfunction. Semin Hear. 1990; 11: 372-83.

25. Riley GD. Stuttering Severity Instrument for Children and Adults. Austin: Pro Ed; 1994.

26. Northern JL, Downs MP. Audição em crianças. 3. ed. São Paulo: Manole; 1989.

27. Jerger J. Clinical experience with impedance audiometry. Arch Otolaryng. 1970; 92:311-24.

28.Auditec. Evaluation manual of pitch pattern sequence and duration pattern sequence. St. Louis: Auditec; 1997.

29. Andrade CRF. Abordagem neurolingüística e motora da gagueira. In: Ferreira LP, Befi-Lopes DM, Limongi SCO. Tratado de Fonoaudiologia. São Paulo: Roca; 2004. p. 1001-16.

30. Runyan CM, Runyan SE. The fluency rules program. In: Onslow M, Packman A, editors. The handbook of early stuttering intervention. San Diego: Singular Publishing Group; 1999. p. 159-69. 